\title{
Levitation and collection of diamond fine particles in the rf plasma chamber equipped with a hot filament
}

\author{
S. Shimizu ${ }^{1}$, T. Shimizu ${ }^{1}$, W. Jacob ${ }^{2}$, H. M. Thomas ${ }^{1}$, and G. E. Morfill ${ }^{1}$ \\ ${ }^{1}$ Max-Planck-Institut für extraterrestrische Physik, Gießenbachstraße, D-85748 Garching, Germany \\ 2 Max-Planck-Institut für Plasmaphysik, EURATOM Association, Boltzmannstraße 2, D-85748 \\ Garching, Germany
}

\begin{abstract}
We demonstrate the levitation of diamond fine particles in a $\mathrm{H}_{2} \mathrm{rf}$ plasma chamber equipped with a hot filament and heated electrodes. The levitation conditions should be carefully chosen to compensate the strong thermophoretic forces caused by the filament and the electrodes. This levitation technique with the existence of a hot filament can be applied, e.g., for the efficient growth of diamond layers on seed particles injected and levitated in a rf plasma with reactive gases, e.g., $\mathrm{CH}_{4} / \mathrm{H}_{2}$. Additionally, the method for direct capture of levitated particles on a planar substrate was established, which is useful if it is necessary to analyze the particles after the levitation.
\end{abstract}




\section{INTRODUCTION}

Plasma-enhanced chemical vapor deposition (CVD) is a method which enables to design materials at an atomic level by decomposing gas into precursors and it has widely been applied for thin film based science and technologies. When growth conditions are chosen properly, it is possible to produce not only films but also micro/nanoparticles that are of great interests in several fields, e.g., quantum dot devices [1, 2], solar cells [3], and etc. While those particles are, in general, grown directly in the gas phase, it is also possible to deposit layers on the seed particles injected prior to the growth where micrometer size particles are levitated in a plasma due to a balance of several forces, e.g., gravity, electrostatic, thermophoretic, ion drag and others $[4,5]$. Hayashi et al. observed the growth of carbon layers on injected and levitated amorphous carbon particles in $\mathrm{CH}_{4} / \mathrm{H}_{2}$ rf plasmas [6]. One can choose gases and type of seed particles to obtain desirable particles with this method. Among many possibilities for those combinations, it is possible to achieve homoepitaxial growth of diamond on an injected seed diamond particle. Indeed, we observed growth of diamond islands on seed diamond particles using $\mathrm{CH}_{4} / \mathrm{H}_{2}$ rf plasma [7]. Our next step is to enhance the growth further more. For example, it takes about $10 \mathrm{~h}$ to obtain those islands with rf plasma, therefore, existence of another precursor generation source is preferable. For this purpose, our system was extended with a hot filament. It was shown previously that the growth rate of diamond using a hot filament CVD technique is generally higher than that with low temperature rf plasma CVD [e.g., 8]. Additionally, compared with low-temperature rf plasma, it creates high density of atomic hydrogen in $\mathrm{H}_{2}$ gas atmosphere, which is one of the essential factors for CVD diamond growth $[9,10,11]$. Moreover, it provides effective heating of the particles due to heat radiation. In general, the optimum substrate temperature for CVD diamond growth is c.a. 1100-1300 K. However, in our case, the levitated particles have some distance from a heated electrode, which may reduce the temperature of the particles from the optimum value. Therefore, existence of an additional heat source is preferable. The rf plasma is necessary to maintain the particle levitation.

In this paper, the actual diamond growth is not studied, but we demonstrate the levitation of diamond fine particles in the $\mathrm{H}_{2}$ rf plasma chamber equipped with a hot filament. Moreover, the method for direct capturing of the levitated particles is also discussed, which is necessary to analyze the particles after the levitation and growth.

\section{EXPERIMENTS}

Figure 1 shows the schematic of the chamber used in this study. The bottom electrode is driven by rf voltage and the top electrode is grounded. The distance between the electrodes is $6 \mathrm{~cm}$ and their diameters are $10 \mathrm{~cm}$. Heaters are mounted in both electrodes. Diamond fine particles (Nilako, size $=2-4 \mu \mathrm{m}$ ) are stored in the dispenser and poured into the plasma. On the bottom electrode, a particle confinement ceramic ring is placed to modify the sheath electric field so that particles are confined above the inside region of the ring. The inner diameter of the ring is $15 \mathrm{~mm}$ and the outer diameter is $25 \mathrm{~mm}$. The temperature of the hot filament is monitored by a pyrometer (Keller HCW, PV11AF1). A $532 \mathrm{~nm}$ green laser (SHG of YAG laser) illuminates the system for visualizing the levitated particles. They are monitored by a CCD camera (Sony, XCD-X700) which detects the Mie-scattered light from the particles. For the particle collection experiment, a positively biased p-type Si substrate of size c.a. $5 \times 10$ 
mm was used as a collector. It was mounted on a transfer rod and carried over the levitated particles region when they are collected. It is a modified negatively charged fine particles collector system [12]. After collection of the particles, the substrate was imaged by a scanning electron microscope (SEM) (Helios NanoLab 600, FEI) [13].

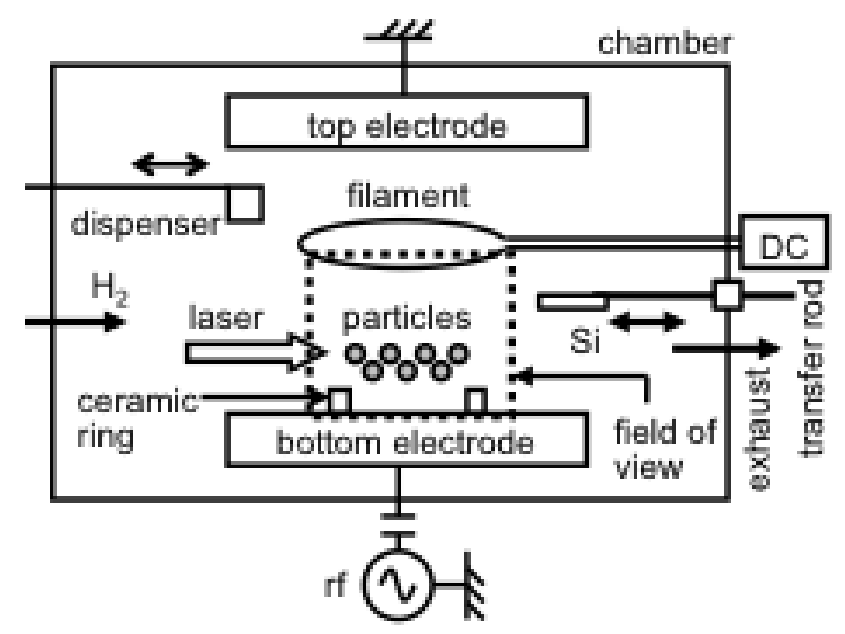

Fig. 1 Schematic representation of the rf plasma chamber used in this study. A tungsten hot filament is installed between the parallel plate electrodes.

\section{RESULTS AND DISCUSSION}

\subsection{Levitation of diamond fine particles}

Diamond fine particles are levitated in the rf plasma chamber equipped with a hot filament. The levitation parameters are the following: $\mathrm{H}_{2}=100 \mathrm{sccm}$, pressure $=300$ $\mathrm{Pa}$, filament temperature $=2300 \mathrm{~K}$, bottom electrode temperature $=1300 \mathrm{~K}$, top electrode temperature $=1100 \mathrm{~K}$, and $\mathrm{rf}$ input power $=50 \mathrm{~W}$. Figure 2 shows an image of levitated particles. In the upper part of the image the hot filament is visible. The diamond fine particles are levitated in the center region of the ceramic ring. Particle levitation was maintained if the pressure was increase up to $1 \mathrm{kPa}$ at the same temperatures. When the pressure exceeds $1 \mathrm{kPa}$, the thickness of the plasma sheath becomes very thin, resulting in no good particle levitation. To obtain steady state levitation, control of the thermophoretic force is one of the important factors. Since the bottom electrode is heated up to $1300 \mathrm{~K}$, fine particles are immediately blown away when there are no counter acting forces. In our system, heating of the filament and the top electrode balance the forces, since the particles are levitated between the bottom electrode and the filament (see fig. 1). Those forces should be applied as uniform as possible in order not to disturb the levitation. Thus, the shape of the filament is also important. If it is a straight line particles are blown away immediately. Therefore, a ring shaped filament was chosen and placed over the outer region of the confinement ring to obtain thermophoretic force directing towards the center region of the ring. In addition, the top electrode which is located above the filament is heated up to $1100 \mathrm{~K}$. The procedure to obtain steady state levitation starting from RT to the high temperature region is the following. First, $\mathrm{H}_{2}$ rf plasma is ignited at RT, then the seed particles are injected form the dispenser. Some of the injected particles are trapped in 
the sheath and levitated in the plasma. At this stage, the total pressure is around 100 $\mathrm{Pa}$. After confirming the levitation, the temperatures of the electrodes and the filament are increased gradually. Since the particles are levitated close to the bottom electrode, their position moves up when the bottom electrode is heated. They would be blown away if there are no counter acting forces. Heating of the filament and the top electrode pushes the particles back down. In addition to the temperature increase, the total pressure is increased from $100 \mathrm{~Pa}$ to $300 \mathrm{~Pa}$ to reduce the plasma sheath thickness. This lowers the levitation height as well. According to the balance of those forces, the particles are finally levitated in the confinement region in steady state.

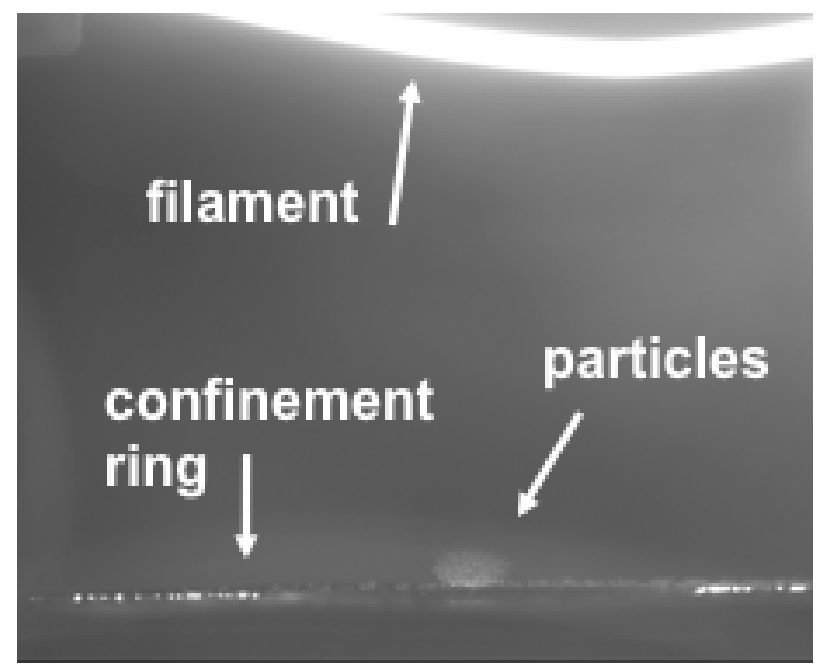

Fig. 2 Levitated diamond fine particles in a $\mathrm{H}_{2} \mathrm{rf}$ plasma with the hot filament. The conditions are: filament temperature $=2300 \mathrm{~K}$, bottom electrode temperature $=1300 \mathrm{~K}$, top electrode temperature $=1100 \mathrm{~K}$, total pressure $=300 \mathrm{~Pa}, \mathrm{H}_{2}=100 \mathrm{sccm}$, rf input power $50 \mathrm{~W}$.

\subsection{Particle collection method}

If layers are deposited on the seed particles, they should be collected to be analyzed. One way to collect them is to put them down on the bottom electrode by controlling the thermophoretic forces. For example, one can switch off the bottom electrode heater with keeping the temperatures of the filament and the top electrode. The $\mathrm{rf}$ plasma should be maintained to keep levitation. It takes roughly 10 minutes until the bottom electrode temperature decreases to below c.a. $800 \mathrm{~K}$. The particles are pushed onto the bottom electrode due to this temperature gradient when the rf plasma is turned off. Then, the particles can be collected after opening the chamber. However, one problem exists with this method. At the beginning of the growth experiment, the seed particles are supplied from the dispenser after igniting the rf plasma at room temperature. We confirmed that roughly one half of them levitate and the others fall onto the bottom electrode. Therefore, when the levitated particles are collected in the way described above, they are mixed with those that have fallen down onto the bottom electrode in the beginning of the experiment. However, the particles on the electrode have experienced significantly different growth conditions, in particular the temperature, from that of the levitated particles. Therefore, it is necessary to capture the levitated particles after growth directly from the gas phase.

Pioneering work for particle collection has been published by Sato et al. using a tube 
as particle collector [12]. This technique is quite efficient to remove unwanted dust particles created in a plasma. We have modified this technique for our purpose and use a flat substrate as collector. This allows an easier analysis of the collected particles. Figures 3 (a)-(d) show a sequence of the particle collection process. (a) A ptype Si substrate (collector) is installed on a transfer rod. It is in a remote position during the plasma treatment of the particles. (b), (c) After plasma treatment it is transferred over the particles levitation region. At pressures lower than about $300 \mathrm{~Pa}$, a bright "spot plasma" appears around the positively dc-biased $(+50 \mathrm{~V})$ collector. When it approaches the particles they are attracted by the spot plasma because they are negatively charged, and levitated just below the collector. (d) Finally, they attach to the collector if the rf power is decreased from 50 to $0 \mathrm{~W}$. In figures 4 (a)-(c), the final phase of the collection is shown, which is the operation between figs. 3 (c) and (d). In each frame the particle tracks are recorded when the shutter is opened $(=11$ $\mathrm{ms}$ ). Those flames are in time sequence (from (a) to (c)). The images are digital processed from the originals with negative filter and un-sharp mask filter for easier view of the particle tracks. The lateral black line shows the bottom surface of the collector. One can see that the particles are attaching to the collector as the rf power is reduced. Indeed, the collected particles are observed on the collector as shown in figure 5 which is the SEM image of its surface after the particle collection procedure.

Maintaining a thermal gradient towards upper direction is important to collect particles on the collector. Because even though the particles are trapped by the spot plasma, the charge of the particles disappears when the rf plasma is switched off, and they will not attach the collector but fall down. To demonstrate the effect of the temperature gradient, the same experiment was performed at RT. Figures 6 (a)-(d) show the results. When transferring the positively biased collector, the particles are levitated below it. Then the rf input power is gradually reduced from $12 \mathrm{~W}$ to $0 \mathrm{~W}$ (from (a) to (d)). One can see that the particles do not attach to the collector but fall down when the rf plasma is switched off (d). The result shows that the attachment of the particles on the collector shown in fig. 3 and fig. 4 is due to thermophoretic force which is estimated in the next section.

Moreover, to see the effect of the potential of the collector, the same experiment was performed at RT with the collector having a floating potential. In this case, the particles fly away from the confinement region as the collector approaches, which is due to the repulsion force between the negatively charged particles and the collector. 

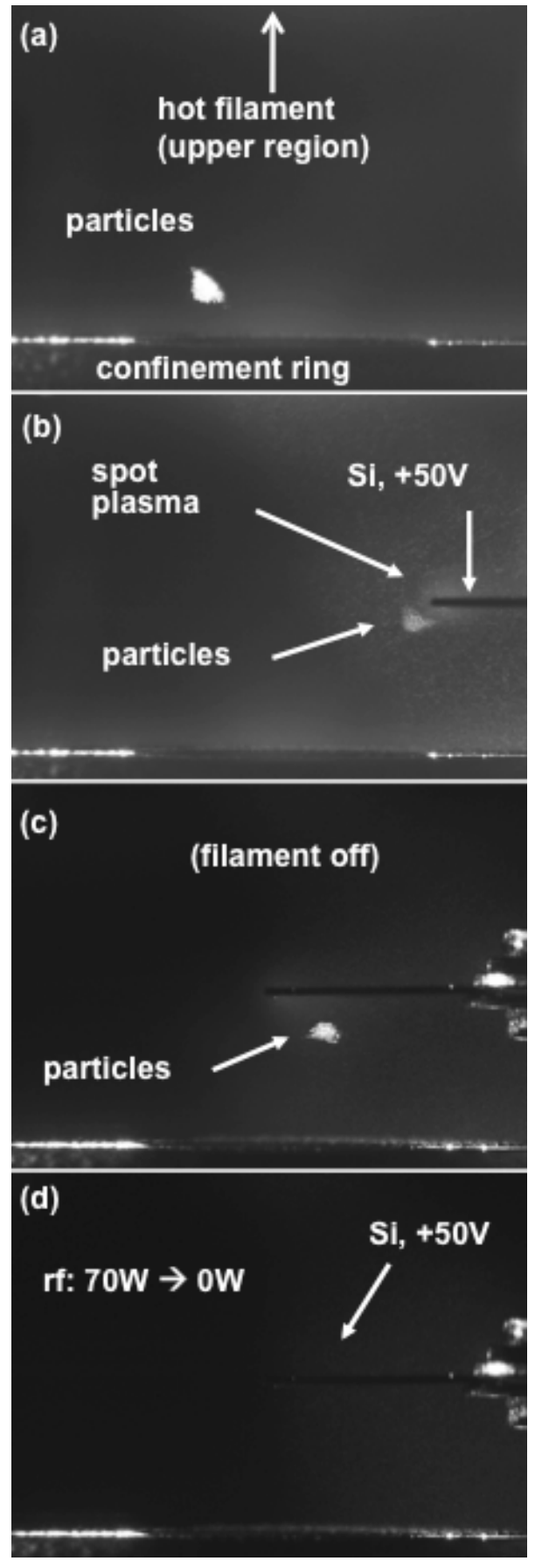

Fig. 3. Sequence of collection of levitated particles with the collector. (a) A p-type Si substrate is used as collector and is installed on a transfer rod. It is in a remote position during the plasma treatment of the particles. (b), (c) It is transferred over the particle levitation region. A bright "spot plasma" appears around the positively dc-biased. When it approaches the particles, they are attracted by the spot plasma. (d) The particles attach to the collector if the rf power is decreased from $50 \mathrm{~W}$ to $0 \mathrm{~W}$. 


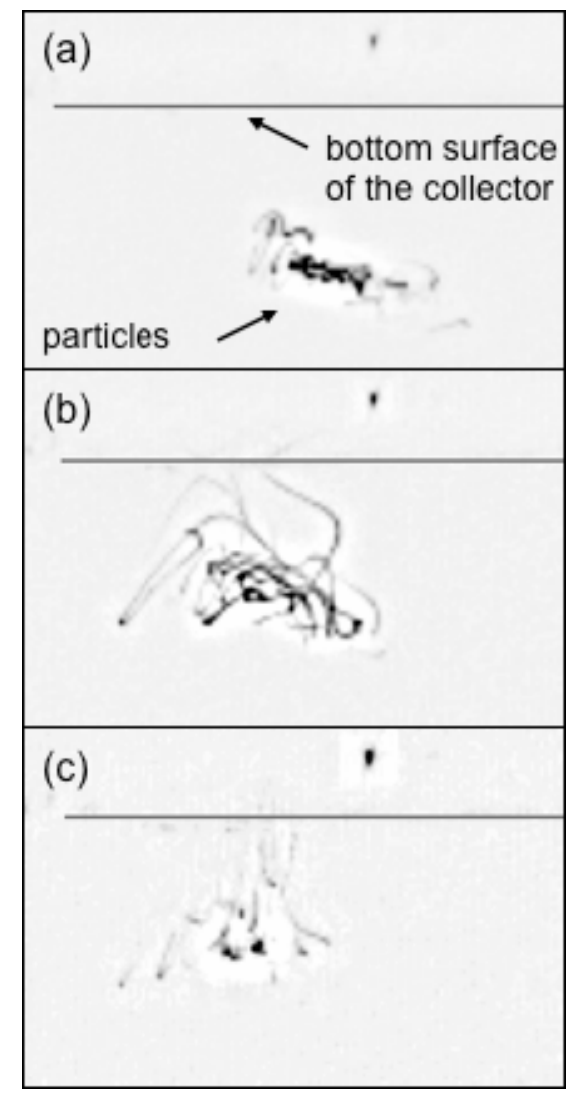

Fig. 4 The final phase of the particle collection. In each frame the particle tracks are recorded when the shutter is opened $(=11 \mathrm{~ms})$. Those frames are in time sequence (from (a) to (c)). The images are digital processed with negative filter and un-sharp mask for easier view. The lateral black line shows the bottom surface of the collector.

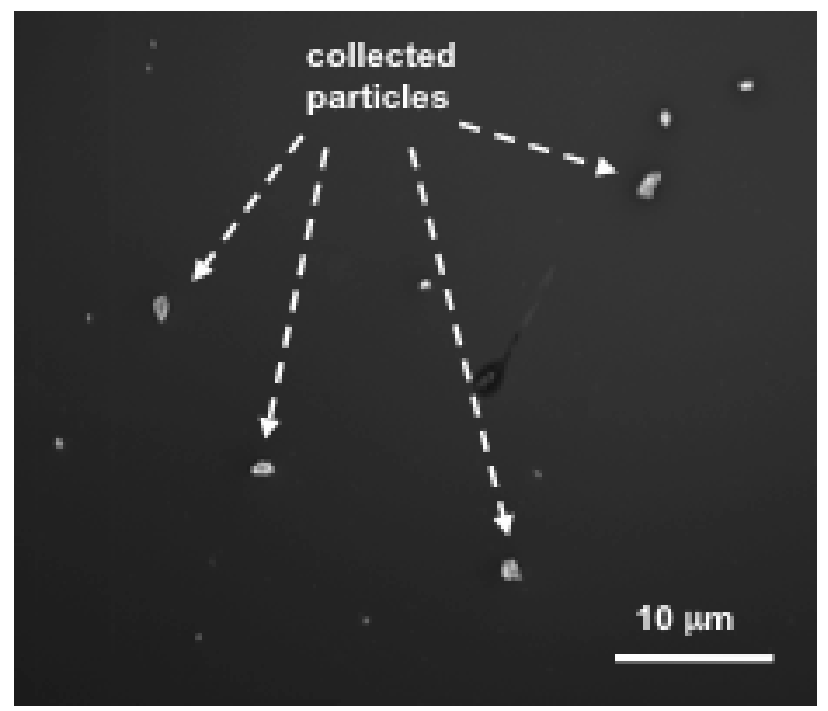

Fig. 5 SEM image of the surface of the particle collector used in fig. 3 and fig. 4. 


\subsection{Estimation of the thermophoretic force}

Here, we estimate the thermophoretic force $\left(F_{\text {th }}\right)$ acting on the particles when they are collected. Since there is basically no plasma during the attachment of the particles to the collector, electrostatic force and ion drag force are not taken into account. To simplify the calculation, the sphere shape diamond particle is considered although the used diamond particles are in edge-like structures. On the other hand, surface area of a particle determines the thermophoretic force acting on it, therefore, it is not very far from the reality in an order estimation level. We take the particle radius $\left(r_{\mathrm{p}}\right)$ of 1.5 $\mu \mathrm{m}$. Thermophoretic force is calculated by the following equation [4].

$$
F_{t h}=-\frac{8}{3} \frac{r_{p}^{2}}{v} \Lambda \frac{d T}{d x}
$$

where $v=(8 \mathrm{kT} / \pi \mathrm{m})^{1 / 2}$ is thermal velocity of the gas atom with the mass of $m, \Lambda$ is coefficient of heat conductivity and $\mathrm{d} T / \mathrm{d} x$ is temperature gradient. The $m$ of $\mathrm{H}_{2}$ is $3.3 \times 10^{-27} \mathrm{~kg}$, and let us assume the gas temperature $(T)$ of $400 \mathrm{~K}$, then, $v=2.0 \times 10^{3}$ $\mathrm{m} / \mathrm{s}$. Next, $\Lambda$ is given by,

$$
\Lambda=2.4 \frac{\eta c}{m}
$$

where $c=3 k / 2$ is the specific heat per atom and the $\eta$ is the shear viscosity of the gas which is given by,

$$
\eta=0.553 \frac{\sqrt{m k T}}{\sigma}
$$

where $\sigma$ is the gas kinetic cross section for atom scattering. The $\sigma$ of $\mathrm{H}_{2}$ is $2.0 \times 10^{-19}$ $\mathrm{m}^{2}$ at the energy of $0.1 \mathrm{eV}$ [14]. Therefore, it contains slight error in our case where the gas temperature is around $0.034 \mathrm{eV}$. However, simple extrapolation of the capture cross-section curve to a lower energy region gives slightly higher $\sigma$, which is the increase at most within factor three. Therefore, let us take the $\sigma$ value shown above, and we obtain $\eta=1.2 \times 10^{-5} \mathrm{Ns} / \mathrm{m}^{2}$ and $\Lambda=0.18 \mathrm{~W} / \mathrm{Km}$.

When the particles are collected, the filament is turned off and the bottom and the top electrodes are kept at $1300 \mathrm{~K}$ and $1100 \mathrm{~K}$, respectively. The collector is transferred in between the two electrodes and the particles are levitated just below the collector. Here, at first, we calculate $F_{\text {th }}$ under the two extreme conditions: (a) under the thermal gradient between the bottom electrode and the collector with neglecting the effect from the top electrode, and (b) under the thermal gradient between the bottom and the top electrode with neglecting the existence of the "cold" collector. 


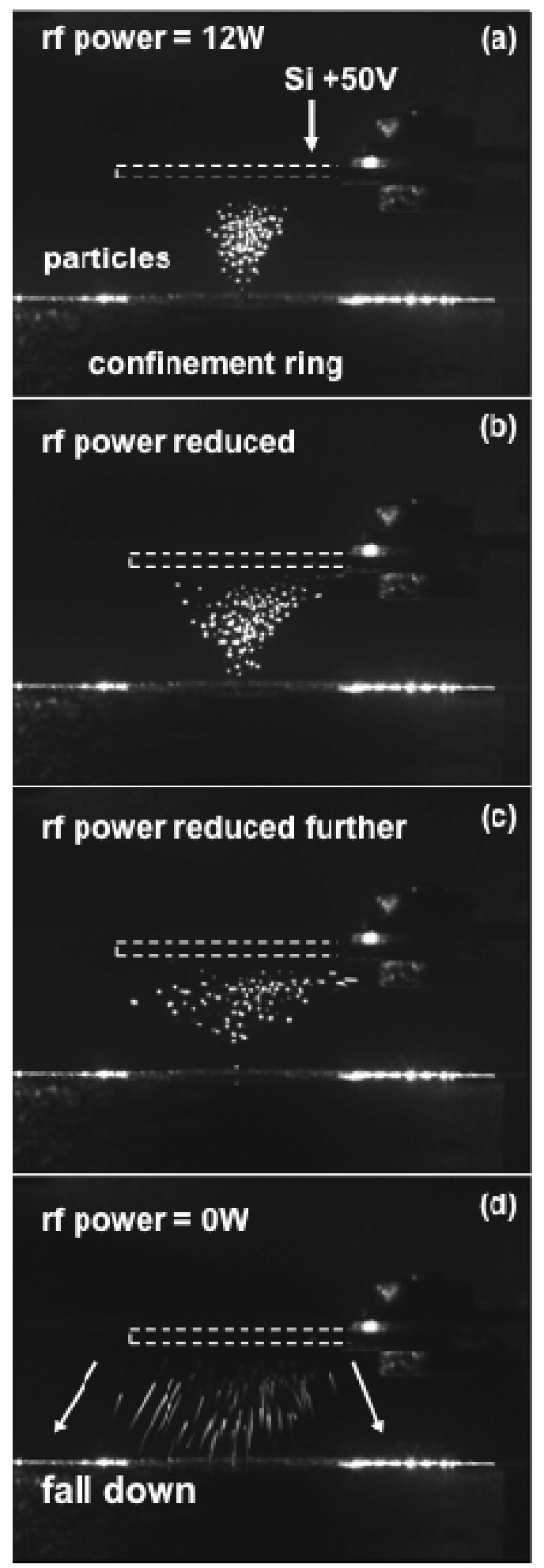

Fig. 6 The sequence of the particle collection at RT. As transferring the positively biased collector, the particles are attracted and levitated below the collector. Then the rf input power is gradually reduced from $12 \mathrm{~W}$ to 0 $\mathrm{W}$ (from (a) to (d)). One can see that the particles do not attach to the collector but fall down when the rf plasma is switched off $(d)$. 
Case (a): during the particle growth, the collector is placed in a remote position, and it is transferred to the electrode region to collect particles. Therefore, we assume the temperature of the collector to be same as the gas temperature $(400 \mathrm{~K})$. The distance between the bottom electrode and the collector is c.a. $2 \mathrm{~cm}$. Therefore, $\mathrm{dT} / \mathrm{d} x=(1300$ $-400) / 0.02=5 \times 10^{4} \mathrm{~K} / \mathrm{m}$. Thus, we obtain

$$
F_{\text {th }}(\text { bottom-collector })=3 \times 10^{-11} \mathrm{~N} \text {. }
$$

Case (b): The thermal gradient between the bottom and the top electrode placed with the distance of $6 \mathrm{~cm}$ is: $\mathrm{dT} / \mathrm{d} x=(1300-1100) / 0.06=3 \times 10^{3} \mathrm{~K} / \mathrm{m}$. Therefore we obtain

$$
F_{\text {th }}(\text { bottom-top })=2 \times 10^{-12} \mathrm{~N} \text {. }
$$

Since the thermal effect from the top electrode heater on the particles levitated below the collector is partially hidden in a short time range, it is reasonable to consider that the actual $F_{\text {th }}$ stays between the two values. This is a simple estimation of the termophoretic force in a lateral direction, and we do not go into details for the gas convection effect that exists in our system.

Next, we consider the gravity force $\left(F_{\mathrm{g}}\right)$ acting on a particle. The $F_{\mathrm{g}}$ of a sphere particle with the mass $m_{\mathrm{p}}$ is calculated as

$$
F_{g}=m_{p} g=\left(\frac{4}{3} \pi r_{p}^{3} d\right) g
$$

where $g$ is gravity constant. Using the mass density $(d)$ of diamond $\left(3.5 \mathrm{~g} / \mathrm{cm}^{-3}\right)$, we obtain

$$
F_{\mathrm{g}}=4.8 \times 10^{-13} \mathrm{~N}
$$

As one can see, the thermophoretic force is larger than the gravity force. If the plasma exists with the collector (see figs. 3 (b) and (c)), the negatively charged particles are trapped by the potential of the spot plasma produced around the positively biased collector. The related effects are studied in details in the former works $[15,16]$. The trapped particles do not attach to the collector even under the strong thermophoretic force, which is due to the plasma and the sheath potential. When the plasma is tuned off, the electrostatic force disappears and the particles are pushed upward by the thermophoretic force.

\section{CONCLUSIONS}

We confirmed the levitation of diamond fine particles in a $\mathrm{H}_{2}$ rf plasma chamber equipped with a hot filament at the high electrode temperature range of around 1300 $\mathrm{K}$. To obtain successful levitation it is important to control thermophoretic forces caused by the heated electrodes and the filament. Additionally, the method for direct capture of levitated particles was established, which is necessary to analyze the particles after levitation and, in some cases, growth. To collect particles, electrostatic force and thermophoretic force are used. 


\section{ACKNOWLEDGEMENT}

The authors would like to acknowledge S. Lindig, M. Balden, G. Matern, and C. Linsmeier in Max-Planck-Institut für Plasmaphysik, EURATOM Association for the SEM measurement.

\section{REFERENCES}

[1] A. Bouchoule and L. Boufendi, Plasma Sources Sci. Technol., 2, 204 (1993).

[2] T. Kamiya, K. Nakahata, Y. T. Tan and Z. A. K. Durrani, I. Shimizu, J. Appl. Phys. 89, 6265 (2001).

[3] M. Green, "Third generation photovoltaics" (Springer-Verlag Berlin Heidelberg, 2003).

[4] H. Rothermel, T. Hagl, G. E. Morfill, M. H. Thoma and H. M. Thomas, Phys. Rev. Lett. 89175001 (2002).

[5] V. E. Fortov, A. G. Khrapak, S. A. Khrapak, V. I. Molotkov, O. F. Petrov, Physics Uspekhi 47447 (2004).

[6] Y. Hayashi and K. Takahashi, Jpn. J. Appl. Phys. 364976 (1997).

[7] T. Shimizu, W. Jacob, H. Thomas, G. Morfill, T. Abe, Y. Watanabe and N. Sato, Thin Solid Films 506-507 652 (2006).

[8] Y. Tang, and D. M. Aslam, J. Vac. Sci. Technol. B 231088 (2005).

[9] H. Umemoto, K. Ohara, D. Morita, Y. Nozaki, A. Masuda and H. Matsumura, J. Appl. Phys. 911650 (2002).

[10] L. L. Connell, J. W. Fleming, H. N. Chu, D. J. Vestyck jr., E. Jensen and J. E. Butler, J. Appl. Phys. 783622 (1995).

[11] S. S. Lee, D. W. Minsek, D. J. Vestyck, P. Chen, Science 2631596 (1994).

[12] Y. Kurimoto, N. Matsuda, G. Uchida, S. Iizuka, M. Suemitsu and N. Sato, Thin Solid Films 457285 (2004).

[13] S. Lindig, M. Balden, V. Kh. Alimov, T. Yamanishi, W. M. Shu, J. Roth, Phys. Scr. T138 014040 (2009).

[14] A. V. Phelps, J. Phys. Chem. Ref. Data 19653 (1990).

[15] T. Antonova, B. M. Annaratone, J. E. Allen, T. Sato, H. M. Thomas, and G. E. Morfill, New J. Phys. 11113023 (2009).

[16] B. M. Annaratone, T. Antonova, D. D. Goldbeck, H. M. Thomas and G. E. Morfill, Plasma Phys. Control. Fusion 46 B495 (2004). 